\title{
Public-access Defibrillation Programs and Out-of-Hospital Cardiac Arrest in Portugal
}

CALDEIRA, P. - Registered Nurse, Emergency Department, Hospital de Cascais, Cascais, Portugal. SANTOS, N. - Registered Nurse, Intensive Care Unit, Hospital Prof. Dr. Fernando da Fonseca EPE, Amadora, Portugal. AMARAL, T. - Registered Nurse, Emergency Department, Hospital de São José, Lisboa, Portugal.

INTRODUCTION: Portugal has undergone a strong development in the number of Automated External Defibrillators (AED) available, through the implementation of legislation, that has forced the creation of AED programs in specific places. AED programs that allow public access to AED's, integrating them with the training of operatives (lay people with basic life support training and AED), with quality monitoring and medical regulation. The Out-of-Hospital Cardiac Arrest (OHCA) survival in Portugal in the last seven years was 7,8\%. Since the beginning of this legislation in 2009, more than 1688 AED's were implemented, all integrated in AED programs.

AlM: Describe the implementation and results in terms of survival of OHCA in public access defibrillation programs in comparison with overall survival of OHCA in Portugal.

METHOD: An observational retrospective study was done, with the analysis of the OHCA data of one of the largest AED programs implementation organization in Portugal, since the beginning of these in 2009, in comparison with the OHCA data of Portuguese Emergency Medical Service database. The Utstein criteria was applied in the data analysis. It was considered statistically significant a p-value less than 0.05 .

\section{SURVIVAL RATE}

35

30

25

20

15

10

5

0

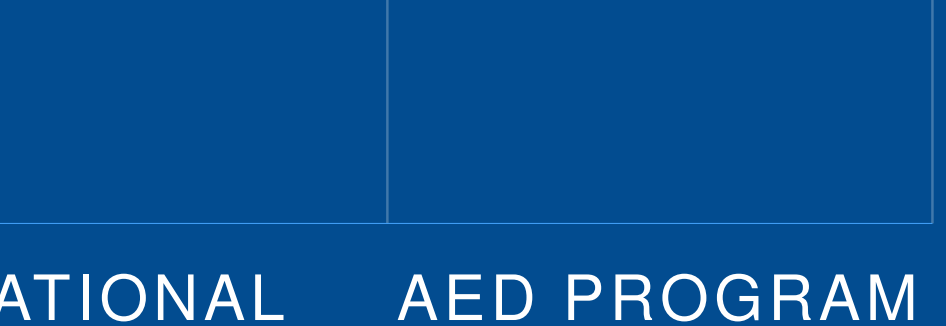

RESULTS: In AED programs we found a survival rate of 30,7\%, in comparison with the 7,8\% (national survival rate of OHCA), AED programs have shown to reduce time to first shock and improve survival in OHCA $(p<0,05)$.

LIMITATIONS: Insufficient data for greater relevance and lack of quality of records derived from the current form of registration.

CONCLUSIONS: We can assume that in presumed cardiac arrests caused survival decreases rapidly as time to defibrillation increases, AED programs have in this study shown that improve survival in OHCA. Portugal needs to continue to focus on strengthening the first three links of the survival chain, the current legislative framework, with the obligation to create AED programs, seems to be the ideal way to guarantee the quality of care for victims of $\mathrm{OHCA}$. 